\title{
Agôn
}

Revue des arts de la scène

$5 \mid 2012$

L'entrée en scène

\section{Hanté en scène : avant le geste et avant le son dans l'interprétation musicale et le théâtre musical et instrumental}

Jean-François Trubert

\section{(2) OpenEdition \\ Journals}

Édition électronique

URL : http://journals.openedition.org/agon/2422

DOI : 10.4000/agon.2422

ISSN : 1961-8581

Éditeur

Association Agôn

Référence électronique

Jean-François Trubert, « Hanté en scène : avant le geste et avant le son dans l'interprétation musicale et le théâtre musical et instrumental », Agôn [En ligne], 5 | 2012, mis en ligne le 25 janvier 2013,

consulté le 02 mai 2019. URL : http://journals.openedition.org/agon/2422 ; DOI : 10.4000/agon.2422

Ce document a été généré automatiquement le 2 mai 2019.

Association Agôn et les auteurs des articles 


\title{
Hanté en scène : avant le geste et avant le son dans l'interprétation musicale et le théâtre musical et instrumental
}

\author{
Jean-François Trubert
}

1 L'entrée en scène est un moment de construction, dont les caractéristiques dépendent de la discipline artistique, mais aussi des contingences de l'œuvre. Objet d'études dans le champ des arts du spectacle, cette question n'a guère été installée dans le domaine de la musicologie et plus particulièrement dans le cadre de l'interprétation, à l'exception des témoignages d'artistes considérés comme reconnus ou des propositions faites dans les textes pionniers de René Leibowitz ${ }^{1}$, lui-même chef d'orchestre et compositeur - mais en évoquant cette question indirectement. Derrière l'idée de l'entrée en scène, il y a celle du passage et du changement qui touche également l'interprète musicien. Il ne construit pas de personnage, mais il respire au rythme d'une intrigue irrationnelle - celle des sons qui lui est imposée par quelqu'un d'autre - le compositeur - auquel il prête son corps. Il s'en accapare le projet et en matérialise l'intention par ses propres gestes: «Toute technique peut être considérée comme une manière d'objectivation de la subjectivité de l'interprète. Les intentions subjectives de l'interprète se traduisent par des gestes $»^{2}$. Avant le jeu, le musicien projette cette intention sur la surface mentalisée du temps à venir, il y forme sa subjectivité, sa propre altérité. Entrer en scène, pour le musicien, pourrait bien être déjà en pensée avant d'être en action, une pensée qui dépend étroitement des caractéristiques de l'œuvre musicale jouée, un moment de potentialités, voire un rituel pour certains.

2 L'entrée en scène peut-elle changer de nature lorsqu'il s'agit d'envisager d'autres répertoires ? Parfois, l'intention du compositeur - qui se manifeste à travers de nouveaux paradigmes formels et structurels - est à réinventer au travers de sa subjectivité propre, et cela pourrait ne pas être sans conséquences pour le geste d'interprétation et pour l'entrée en scène, en particulier dans le champ du « théâtre musical d'avant-garde $»^{3}$ ou encore du 
"nouveau théâtre musical $»^{4}$ et du théâtre instrumental ${ }^{5}$ : un genre que la musique dite savante s'est approprié ${ }^{\prime}$ au moment même où le théâtre remettait en question ses formes dans la période appelée "post-dramatique» par Hans-Thies Lehmann ${ }^{7}$. L'interprète musicien relèverait-il alors un double défi, qui n'est plus si éloigné de celui que relève l'acteur contemporain: mentaliser le temps d'une œuvre plurielle et fragmentaire, incarner un monde émotionnel et corporel multivalent, où le spectacle se construit non seulement par la technicité du son et la maîtrise du geste instrumental, mais aussi par des gestes incidentaux, des paroles, des actes signifiants qui débordent du cadre "sonore" traditionnel ? Ce serait le cas notamment dans les pièces de théâtre musical de Mauricio Kagel, comme Acustica (1970), Match (1964) ou encore Zwei-Mann Orchester (1973). C'est le cas également dans les pièces de Georges Aperghis comme Les Sept Crimes de l'amour (1979), ou Récitations (1978), Machinations (2000), Luna Park (2011), etc.

3 Il semblait pertinent de sonder cette question de l'entrée en scène dans le domaine de l'interprétation musicale, puis de la mettre en regard de la spécificité du geste et de l'avant-geste du théâtre musical et instrumental. L'étude s'attache d'abord à donner un contexte théorique à la réflexion, puis exploite des entretiens réalisés auprès de praticiens de la musique. Ces quelques témoignages ont notamment apporté, et c'est l'objet de notre deuxième chapitre, des éclairages sur ce moment de l'entrée en scène pour le musicien, définissant des étapes particulières, voire spécifiques (puisque liée à une discipline instrumentale particulière) dans le domaine qui nous intéresse. Enfin, confrontant théorisation et témoignages, la question de la relation du geste à la forme de l'œuvre, entre homogénéité et hétérogénéité, est évaluée à travers ce prisme de l'entrée en scène et l'état de concentration qui s'y rattache.

\section{L'interprétation musicale : un geste mentalisé}

Le domaine de l'interprétation musicale constitue un champ d'études musicologiques dont la bibliographie est assez volumineuse ${ }^{8}$. Du fait de son double sens en français (interprétation comme compréhension d'un texte, ou comme acte de représentation) ces études se rattachent autant à l'herméneutique ${ }^{9}$ qu'à l'étude de la représentation ${ }^{10}$, ce que l'on nomme performance studies dans la musicologie anglo-saxonne, terme affilié à la discipline des arts du spectacle - ce dont, entre autres, témoignent les travaux de Richard Schechner ${ }^{11}$ - mais aussi à l'ethnologie et à l'anthropologie. Le mot perform signifie accomplir une action, et si l'on peut remarquer aujourd'hui une tendance à utiliser l'anglicisme performer ${ }^{12}$ pour désigner sans distinction l'interprète, l'exécutant, ou l' agissant, nous retiendrons pour cette étude la distinction qui est faite par Hans-Thies Lehmann: ce qui distingue le performer du comédien, c'est la fiction, absente dans le premier cas : «souvent le comédien du théâtre postdramatique n'est plus le représentant d'un rôle (actor) mais le performer qui offre sa présence sur la scène à la contemplation » ${ }^{13}$. En ceci, interprète musicien et acteur au $\mathrm{XXI}^{\mathrm{e}}$ siècle se rejoignent sur un terrain partagé ${ }^{14}$.

Chaque interprétation musicale est singulière, et ce jeu particulier exprime une subjectivité, ce que Nicolas Donin qualifie de «lecture $»^{15}$, une compréhension du texte musical, malgré son apparente intelligibilité. Ce sens herméneutique va être soumis à un questionnement par René Leibowitz, qui relie l'analyse à l'interprétation musicale, en confiant à la première le soin de relever dans le détail les éléments saillants de cette herméneutique, dont le produit se matérialise ensuite dans l'acte du jeu : les « gestes » de 
l'interprète. Joëlle Caullier, dans deux articles ${ }^{16}$, relie cette expérience à celle du corps ${ }^{17}$, sa matérialité palpable en quelque sorte, coexistant à tout acte interprétatif. C'est dans cet esprit que l'on peut tenter de définir différentes strates constitutives de cette corporéité de l'interprète, qui a été socialement constitué tout en étant le reflet d'une intentionnalité subjective :

La sonorité d'un musicien, c'est la matérialisation sonore de son propre corps, d'un corps qui a été façonné par une expérience vécue; elle n'est pas seulement le produit d'une technique ou d'une facture instrumentale particulière, sinon tous les musiciens issus d'une même école ou jouant le même instrument auraient la même qualité sonore ; elle est surtout le produit de la vie dans sa singularité. ${ }^{18}$

Dans le cas de la musique, l'interprète rend sensible un certain rapport au monde à travers ses gestes propres médiatisant ceux d'un autre - le compositeur. C'est ce moment de contact, ou d'appropriation, qui donne sa substance à l'acte interprétatif ${ }^{19}$. Cette vie mentale et affective se prépare dans le moment de l'entrée en scène, car elle ne peut exister sans l'intention. Celle de l'interprète musicien se pense en relation avec le corps, et avec la manière dont on pense ce corps entrant en relation à la pièce elle-même. Ce moment se constitue dans un rapport de projection informée de soi.

Ces questions d'ordre théorique nous serviront d'assises durant tout notre commentaire pour aborder l'entrée en scène, que l'on a tenté d'explorer à travers quatre entretiens. Nous avons pu tout d'abord interroger Whilhelm Bruck, guitariste, qui a collaboré dès les années 70 avec Mauricio Kagel et a été l'interprète historique de la création de certaines de ses œuvres. L'entretien a été réalisé à l'occasion de la réalisation à Bâle au musée Tinguely en avril 2011 de la pièce de théâtre instrumental Zwei-Mann Orchester (1973) de Mauricio Kagel, à la création de laquelle Bruck participa en 1973. Le deuxième témoin est Gérard Siracusa, percussionniste et compositeur, qui a collaboré avec l'ensemble musique vivante de Diego Masson, avec Joëlle Léandre ainsi qu'avec l'ATEM ${ }^{20}$. Il a réalisé de nombreuses performances de théâtre musical et d'improvisation, et a eu la gentillesse de bien vouloir répondre à nos questions. Le troisième témoin est Philippe Serra, premier timbalier solo de l'Orchestre Philharmonique de Nice, qui s'est prêté au jeu avec beaucoup de bienveillance, et dont l'entretien très éclairant est reproduit dans l'enquête. Enfin, un dernier entretien a été réalisé lors d'un travail d'accompagnement du récital de Florian Poirier - aujourd'hui professeur assistant de la classe de percussion du Conservatoire à Rayonnement Régional de Nice - en avril et mai 2012. Celui-ci souhaitait l'insertion d'une pièce de théâtre musical dans un répertoire de récital de concert, et le choix s'est porté sur une esquisse inédite de la pièce Match de Mauricio Kagel, comprenant de nombreuses indications textuelles avec notamment un descriptif d'entrée en scène ( $c f$. infra). Cette étude, volontairement concentrée sur un panel restreint d'interprètes, essentiellement percussionnistes, s'attache moins à la recherche d'une définition exhaustive qu'à l'élargissement des perspectives ${ }^{21}$. Ceci nous a permis de définir des espaces et des moments de l'entrée en scène du musicien, d'en voir les particularités lorsqu'il s'agit de théâtre musical et quels enjeux cela pose pour l'interprétation, et enfin de poser la question du statut de l'interprète musicien au moment du jeu.

\section{Les zones frontières multiples : espaces et moments}

8 Selon Béatrice Picon-Vallin, l'entrée en scène est la redéfinition d'une zone comme zone du jeu : 
sur le sismographe du théâtre, le statut du comédien oscille selon les zones qu'il doit investir - les planches de la scène, un morceau de réel fictionné, une machine à jouer, un lieu où apparaître et revenir en évoquant d'autres re-venants. ${ }^{22}$

9 L'entrée en scène du musicien se fait par rapport à un espace figé qui lui préexiste : le plateau qui contient ou non un instrument, et qui en général s'offre déjà à la vue du public, vide, ou avec les instruments seuls, appendices attendant de prendre vie. Gérard Siracusa désigne comme moment le plus important de l'entrée en scène celui de la "parution sur le plateau » ${ }^{23}$, c'est-à-dire lorsque l'interprète s'offre à la vue du public. Selon Philippe Serra, ce moment s'accompagne d'une "prise de conscience de l'environnement » :

[...] surtout lorsque l'on se trouve dans une nouvelle salle. Tout ceci se fait au moment où l'on s'assoit et où l'on s'accorde avec l'orchestre. Il y a toute une ambiance que l'on essaye de capter au maximum, tout en étant concentré et en essayant de positiver le moment où l'on va jouer. ${ }^{24}$

10 Il s'exerce en quelque sorte un contact, un contact du lieu et de l'humain, qui s'accompagne d'un état de l'interprète. Ce qui doit advenir est en quelque sorte déjà là, mais de manière inconsciente : «Entrer en scène, c'est livrer plus de signes que l'acteur peut avoir conscience d'en émettre, et, pour le spectateur, c'est recevoir plus de signes qu'il ne peut analyser $»^{25}$.

11 L'autre moment de l'entrée en scène est celui du contact avec l'instrument, ce que Gérard Siracusa nomme fort justement «la mise en place corporelle dans le dispositif instrumental». Or, ce moment peut varier et, chronologiquement, il peut avoir été préparé en amont. Dans le cas des instruments de percussions, l'instrumentiste doit venir s'accorder avant le lever de rideau, en particulier pour les timbales. Philippe Serra insiste sur cette nécessité d'aller dans la salle, avant l'entrée du public. Selon lui, l'entrée en scène s'étend à cette préparation :

Personnellement, je m'habille et ensuite je vais m'accorder le plus vite possible pour être sûr que les timbales sont bien réglées, que les baguettes sont bien posées. Ensuite on ressort et on attend l'entrée en scène dans les coulisses, on s'échauffe les poignets, on discute un petit peu, pour se décontracter, cela se déroule dans plusieurs lieux.

12 Le fait de pouvoir régler l'instrument semble être un paramètre important comme préalable à l'entrée en scène. En 2011, Whilhelm Bruck se plaignait par exemple de ne pas avoir eu assez de temps pour préparer le dispositif instrumental ${ }^{26}$. Gérard Siracusa insiste également sur ce point en relation avec l'état de décontraction nécessaire avant le jeu :

Je travaille toujours à ce que [la mise en condition de jeu] demande le moins de temps de préparation possible. Dans l'idéal, elle devrait pouvoir être instantanée. Souvent, il faut réactiver la mémoire auditive comme gestuelle de la pièce à jouer, par relecture d'extraits, récapitulation de suites gestuelles, révision mentale lorsque l'on joue par cœur. Ce temps peut être plus ou moins long. Cela dépend de la fatigue, du stress éventuel, de l'endroit où l'on joue... Je constate que la prise en possession spatiale d'un lieu quelques heures en amont permet souvent la bonne disponibilité en seulement quelques minutes de concentration pure avant l'entrée en scène.

13 Entrer en scène nécessite une préparation mentale pour s'approprier une sonorité et un nouvel espace, tout en passant au travers de plusieurs zones: préparation de l'instrument, retour en loges, parution sur le plateau, «mise en place dans le dispositif instrumental». Cette multiplicité des espaces renvoie à une multiplicité de moments, dont la plupart coïncident avec les étapes décrites et peuvent être rassemblés sous une 
catégorie : le moment du plateau, cette succession d'aller-retour et de préparation jusqu'à la parution à la vue du public, suivi du jeu.

Puis, il y a ce que nous pourrions appeler le moment musical de l'entrée en scène, qui est lié tout simplement à l'écriture, à l'œuvre. Elle met en jeu des phénomènes d'ordre spectaculaire et dessine de véritables lignes de forces - sorte de dramaturgie sans signification ${ }^{27}$ - où apparaissent des phénomènes localisés. On me pardonnera d'évoquer ici des moments parfaitement ancrés dans l'histoire de la musique : comme l'acte 3 des Walkyries de Wagner; ou encore cette entrée en scène particulièrement frappante du thème de la Cinquième Symphonie de Beethoven; ou les entrées obstinées de la figure mélodique du Boléro de Ravel.

Dans une œuvre pour ensemble, le moment musical de l'entrée en scène individualise physiquement les instrumentistes, qui, lorsqu'ils jouent la mélodie principale, entrent. À l'écriture musicale d'un thème correspond alors une information visuelle qui concerne l'instrumentiste qui joue. À l'opéra, la partition guide l'apparition de tel ou tel chanteur ; à l'orchestre, elle individualise le soliste dans un concerto; ou on peut considérer avec Philippe Serra, que les premières mesures d'une pièce constituent cette entrée en scène par leur caractère même :

Par exemple la première de Brahms : c'est une entrée en scène difficile, même si on l'a jouée plusieurs fois ${ }^{28}$, parce que dès les premières mesures elle développe, elle dégage une puissance dramatique assez incroyable [...] D'emblée les timbales donnent le ton d'un élan très, très dramatique et lyrique, et il faut veiller à sa sonorité sans être crispé sans être agressif, tout en déployant une force énorme. Dès les premiers instants, pour la sonorité, le caractère, la densité, il ne faut pas être agressif et il faut être très concentré. Voilà une entrée en scène très particulière.

\section{Le moment musical de l'entrée en scène dans le théâtre musical : le geste sous contrôle}

Dans le cadre du théâtre musical d'avant-garde ou contemporain, c'est-à-dire les pièces qui sont composées à partir de la fin des années 1950 par des compositeurs comme Mauricio Kagel, Georges Aperghis, mais aussi Vinko Globokar ou encore Jean-Pierre Drouet, moment de plateau et moment musical de l'entrée en scène sont en fait confondus. Gérard Siracusa rappelle que « dans le cas du théâtre musical [...] l'entrée sur le plateau coïncide avec l'entrée en jeu ${ }^{29}$. Car celle-ci est en général décrite dans la partition, ce qui s'apparente bien souvent à des didascalies - comme pour Sonant 1960/... (1960) de Mauricio Kagel. Dans un autre registre, Philippe Serra partage son expérience à propos de la pièce Martial Tribes (1995) d'Emmanuel Séjourné où « il y a une mise en scène décrite, mais l'entrée des interprètes est complètement libre et spontanée [...] cette impression de liberté en entrant sur scène c'était assez exaltant et assez nouveau ». L'interprète s'expose, il devient décideur, et choisit ses propres mouvements, même s'ils sont laissés libres. Dans les Sept Crimes de l'amour (1979) de Georges Aperghis, les trois interprètes sont déjà sur scène, et la première section de la pièce qui en compte sept s'ouvre sur une action qui a débuté hors de la vue des spectateurs, qui la surprennent ${ }^{30}$. Dans la pièce Music Walk (1958) de John Cage ${ }^{31}$, qui a inspiré historiquement l'idée de théâtre instrumental en Allemagne ${ }^{32}$, les interprètes se livrent à un déambulatoire pour actionner un dispositif instrumental hétéroclite disposé dans toute la salle. La situation est ici renversée car l'entrée en scène n'existe pas, ou plutôt le mouvement de l'entrée est confondu avec le mouvement de l'action musicale - faire émettre un son par un objet - 
action qui noie l'entrée en scène par sa répétition en lui ôtant son caractère de rituel. Autant de pièces qui jouent avec cette catégorie, avec ce rituel du spectacle.

Dans le théâtre instrumental de Mauricio Kagel, ce ne sont pas les interprètes qui entrent en scène, mais les instruments qui font leurs entrées, comme dans Acustica (1969-70), ou dans Zwei-Mann Orchester (1973). Il y a un renversement de l'effet visuel par l'aspect iconoclaste des instruments inventés et originaux. Soit leur mode de jeu surprend, soit ils surgissent au fur et à mesure du spectacle - au même titre que les personnages d'une pièce de théâtre feraient leurs entrées successives par le truchement des portes ou des placards de la scène. La section Repertoire de Staatstheater (1971) en est symptomatique : un jeu de panneaux astucieusement disposés cache les interprètes pour ne laisser apparaitre parfois qu'un instrument ou qu'un objet: l'ensemble de cette section est une suite d'entrées en scène successives. Dotés d'une sonorité spécifique et d'une modalité de jeu humoristique ou inattendue, l'effet visuel s'ajoute à la dimension sonore pour imposer une sorte d'«effet d'étrangeté $»^{33}$. En forçant le trait, nous pourrions dire que c'est l'interprète qui est instrumentalisé, en particulier dans Zwei-Mann Orchester, et l'instrument qui s'impose comme objet montré, soudain animé et doué de vie, entrant en scène à la vue du public. Mais cet instrument meurt lorsque l'on cesse d'en jouer, plongeant l'auditoire pris au piège dans une sorte d'angoisse de la perte : humour noir ! L'instrument, le son et son geste d'émission sont alors mis au premier plan et construisent tous les éléments du spectacle, tout comme les instruments qui sont disposés dans le cadre de la page du Theatrum Instrumentorum de Michael Praetorius ${ }^{34}$ constituent ce qui est montré.

Un exemple encore plus concret peut illustrer cette problématique de l'entrée en scène pour l'interprète musicien dans le cadre du théâtre instrumental. Pour son récital ${ }^{35}$, Florian Poirier avait décidé de donner à son récital une tonalité proche d'un esprit théâtre musical contemporain en une sorte de mise en scène, exploitant pour cela la situation de certaines pièces (duo, disposition des instruments face-à-face, disposition scénique des claviers). Le répertoire était constitué des pièces suivantes, entre autres : festa per due (1999) de Nicolas Martynciow, Reflexion on the nature of water (1986) de Jacob Druckman, Kerberos (1990) de Sydney Hodgkinson, la huitième pièce La Marche des Pièces pour timbales (1966) d'Elliot Carter et Rebonds A (1987-89) de Xenakis ${ }^{36}$. Florian Poirier souhaitait trouver un élément de transition avant d'aborder la pièce Kerberos situé entre la fin du premier tiers du récital et sa moitié. Or, il se trouve que Mauricio Kagel avait réalisé un projet inédit de mimodrame intitulé Match, mimodrama für Ratsch und Darsteller (Match, mimodrame pour Crécelle et interprète) ${ }^{37}$, projet qui évoluera ensuite vers la pièce de théâtre instrumental éponyme plus connue, pour deux violoncelles et un percussionniste ${ }^{38}$. Cette esquisse comprend une suite de descriptions textuelles de différentes actions et de gestes avec une frise temporelle, que l'on peut résumer comme étant le combat d'une main contre l'autre pour la domination d'une crécelle, afin de l'actionner sur une caisse claire en un geste final. Cela se présentait très bien dans le projet de récital de Florian Poirier, car la pièce qui suivait, Kerberos, utilise exclusivement la caisse claire ${ }^{39}$. Juste avant, il a donc été décidé d'insérer ce mimodrame kagélien, dont les premiers mots sur le brouillon conservé à la Fondation Sacher se présentent ainsi :

$1-$

$20 "$

Entrer depuis la gauche. La crécelle est cachée.

Rester debout soudain, presque dos au public, et faire une courte pause.

(L'instrument n'est pas visible) 
2-

$12 "$

a. La tête ne bouge pas.

La crécelle dans la main droite, allonger le bras droit si bien que tous les spectateurs puissent voir l'instrument. La crécelle se balance encore au bout sans que la langue puisse tourner autour de la dent...

b. ....de manière très régulière (pas de son! seulement près du bruit) observer le mouvement

de la crécelle (le corps). ${ }^{40}$

Ce média ne peut être affiché ici. Veuillez vous reporter à l'édition en ligne http:// journals.openedition.org/agon/2422

Cette pièce fonctionne comme une sorte d'extension de l'avant-premier geste, et par là comme une hypertrophie de l'entrée en scène, une entrée en scène dirigée, mise en scène, décrite par de multiples didascalies avant le tout premier son. Celui-ci en est à la fois le but et la fin, objectif réduit à son strict niveau élémentaire - comme le plan et le cadre des avant-gardes constructivistes, le Carré noir sur fond blanc (1915) de Malevitch, comme le silence de 4'33" (1952) de John Cage. Dans cette esquisse de Match, c'est un son résultant de la réunion d'un souffle et d'un geste qui signe la fin de la pièce. Celle-ci constitue un intéressant archétype de la situation type du théâtre musical et instrumental : le rapport entre le geste d'émission et le son produit.

Mais ceci renvoie à l'intentionnalité de l'interprète et à un paradoxe: alors que des descriptions l'amènent au mouvement corporel et au geste, il perd le contrôle de sa part d'interprète qui lui était dévolue : il ne gère plus de la même façon le "grain » et son intentionnalité subjective, parce qu'il doit y contrôler cette fois-ci le geste producteur.

\section{L'entrée en scène de soi : intériorité et/ou mise en disponibilité}

L'entrée en scène correspond dans tous les cas à une transformation de l'état intérieur commune à beaucoup d'expériences de la performance - au sens premier du terme : «attentif à lui-même [...] un silence intérieur dont l'acteur a besoin pour se concentrer, [...] tout en lui est préparé dans la même décontraction physique que celle du sportif $~^{41}$.

Or on retrouve une proposition similaire décrite par Gérard Siracusa: "La mise en condition de jeu est une mise en disponibilité la plus grande possible du corps et de l'esprit $»^{42}$. De plus, à la question " pensez-vous que certaines pièces musicales nécessitent des états émotionnels particuliers pour les exécuter?», Gérard Siracusa répond : «Je ne vois pas d'autre état que celui de la concentration et de la disponibilité $»^{43}$. Florian 
Poirier, dont nous venons d'évoquer le travail, insistait également sur l'état de concentration nécessaire :

Quand j'arrive je prends 5 à 6 secondes et je respire profondément, je me mets en condition [...] Une fois que je suis rentré, je ne sors plus. C'est-à-dire que le plus difficile c'est vraiment de rentrer, de se mettre dedans. C'est un peu comme rentrer dans une sorte de bulle : il y a une résistance, mais une fois passée, on ne sort plus avant la fin. C'est vraiment rentrer dedans qui est le plus difficile. ${ }^{44}$ espèce de relaxation, de sérénité, pour découvrir une œuvre ou pour la faire découvrir, pour y entrer comme si c'était la première fois qu'on l'interprétait». Dans ces trois témoignages, l'idée d'un passage est récurrente, Jean-Loup Rivière parlera de «frontière »: «Entrer, c'est effectivement un franchissement, le passage d'un monde à l'autre $»^{45}$. Le musicien s'imprègne d'un silence qui est chargé de tous les signes de la salle: «on capte un peu du silence du public, et puis notre propre silence dans l'orchestre, notre propre concentration $»^{46}$. Ce passage vers l'état émotionnel, moment de concentration, est encore très justement défini par M.-P. Lassus :

Avant d'entreprendre sa tâche, l'interprète doit d'abord se mettre en état de disponibilité, dans une sorte de «disparition subjective », qui permet de sentir ce passage du corps statique au mouvement pur. Dans le silence, il crée un espace uni et calme où le corps va puiser pour trouver le « geste juste » issu du plus profond de l'intériorité. Cette disposition subjective qui est propre au musicien sur le point d'attaquer un morceau, est en tout point comparable au duende du cantaor de flamenco, ou à l'intention qui précède l'exécution du trait de pinceau dans l'art pictural chinois. C'est de cette intention que naît le geste juste issu lui-même du corps tout entier. ${ }^{47}$

Cette intention est indispensable, elle est activée par la conscience «imageante» de la pièce musicale - Gérard Siracusa parle de se remémorer les passages de la pièce, de se l'incorporer mentalement - et par l'écoute informée - l'analyse - qui va orienter les choix et les stratégies interprétatives :

C'est important de savoir ce qu'il y a derrière une œuvre, quand on aborde l'écriture de la pièce dans son ensemble. Je regarde souvent la partie de timbale à travers la partie d'orchestre pour bien comprendre l'architecture de la pièce et en faire son analyse. ${ }^{48}$

L'expérience et la connaissance jouent alors un rôle non négligeable dans la préparation de cette intention. Par exemple, Philippe Serra évoque des pièces qui demandent un investissement émotionnel et une mise en condition avant le jeu, à l'entrée en scène :

Quand on joue Xenakis ${ }^{49}$, il faut quand même avoir une charge émotionnelle assez importante juste avant le premier coup. Là, on est un autre avant de jouer la première note. Il faut plus de spontanéité dans le jeu quand on joue une pièce soliste comme cela.

Pour Florian Poirier, qui exécutait Rebonds A de Xenakis lors de son récital, le travail sur la pièce nécessite également une mise en condition très particulière avant le jeu :

je prends l'exemple de Rebonds, si on ne part pas avec une sorte de rage, moi je n'arrive pas à la fin. [...] Quand j'arrive [devant mon instrument] je me mets en condition, surtout pour des pièces comme ça, physiques. ${ }^{50}$

Cette mise en condition est également mentale :

[L'interprète] est quelqu'un d'autre, mais sans vouloir être quelqu'un d'autre; il faut qu'il soit vraiment habité par la musique. C'est l'équilibre le plus dur à trouver. 


\section{interpénétration:}

Il semble évident que pour le musicien les structures musicales de l'œuvre qu'il interprète parlent un langage éloquent. Il en saisit et en actualise le sens au moment où livré à sa partition il est comme possédé par elle, en même temps qu'il la possède. ${ }^{51}$

La préparation de cet état passe par une modification émotionnelle :

car d'un côté, cette partition lui parle d'un crescendo ou d'un decrescendo à faire, et c'est ainsi qu'elle le possède [l'interprète], alors que, de l'autre côté, c'est à l'interprète de posséder cette partition, puisque sans son acte volontaire il n'y aurait ni crescendo ni decrescendo, ni même exécution tout court. L'expression musicale est une praxis ; cela n'exclut évidemment pas l'effort intellectuel qui à tout moment soutient (et peut-être se confond avec) l'impulsion émotionnelle. ${ }^{52}$

Pour autant, il n'y a pas de «mystique » selon les propres termes de Gérard Siracusa. Une des difficultés, dans l'interprétation des pièces de musique contemporaine qui appartiennent au genre du théâtre musical, réside dans leur nature hybride. Non qu'elles demandent des prouesses techniques insurmontables, mais plutôt une concentration des forces dans tous les medium à la fois. Elles rompent en général avec le schéma classique du geste producteur de son, un paradigme qui va du geste au son : Geste/Emission du son, comme nous l'évoquions plus haut pour Match. Le rôle et la liberté de l'interprète peuvent en effet se situer dans ce façonnage du geste, dont le but est de faire entendre ce qui est écrit sur la partition. En effet, selon Philippe Serra :

L'interprète est un professionnel, [...] Il faut qu'il soit juste, c'est-à-dire qu'il ne faut pas qu'il sur-joue ou qu'il ne joue pas. [...] Pour nous les timbaliers c'est très facile de tomber dans le mimétisme, dans des grands gestes qui ne correspondent pas à la sonorité.

Or, dans le théâtre musical et instrumental, il y a justement une orientation du compositeur qui tend à intégrer cette partie d'avant le son - le geste donc - comme élément et paramètre de la composition. D'où une séparation entre geste et son, et une réelle question d'interprétation à évaluer pour l'interprète, qui doit non seulement à présent contrôler le son émis pour qu'il soit « juste», mais aussi le geste producteur, qui peut être soumis à un contrôle total ${ }^{53}$. Whilhelm Bruck dira par exemple qu'il est fondamental de garder une distance avec les événements, et rappelle que Kagel lorsqu'il compose, soumet le moindre geste d'exécution instrumentale à un examen approfondi. C'est en ce sens que Florian Poirier ${ }^{54}$ avait évalué l'enjeu qui se cachait derrière les pièces de théatre musical et instrumental par rapport aux indications textuelles de l'esquisse inédite de Match :

«Parce qu'il y a une composante qui rentre en plus. Tout d'abord, quand on joue sur un instrument, on pense à un aspect technique, à un aspect musical. Ici, on rentre dans ce cadre, parce qu'on a une technique propre par rapport à la crécelle. Il y a des indications musicales pour réfléchir à la manière de faire sonner la pièce, avec la caisse claire sur la fin. Mais en plus, il faut réfléchir intellectuellement à la façon de rendre cela crédible visuellement. Il y a quelque chose en plus quand on joue sur un instrument. Prenons Glenn Gould par exemple: s'il joue tout recroquevilléle ${ }^{55}$, on s'en moque parce qu'il joue divinement. Mais là c'est autre chose : il faut que cela soit crédible visuellement, pas forcément beau, mais avec une posture en plus à défendre $»^{56}$.

En effet, ce geste qui avant n'était pas soumis à un contrôle particulier, du moment que la partition était correctement exécutée, mérite dans des pièces comme Zwei-Mann Orchester 
un changement de conception, une tournure d'esprit différente, comme le précise Whilhelm Bruck: «Je pense que ce qui est important c'est qu'il y ait une connexion inséparable entre le son et le geste. On peut voir et écouter séparément mais cela ne marche pas $»^{57}$. Prenant en exemple une série d'action du début de Zwei-Mann Orchester, Bruck décrit une situation où l'on doit passer d'un instrument à un autre et décrit comment penser le geste de production du son :

On joue par exemple tremolando, avec un archet, touctouc touc touc touc, et puis alors viennent les gouttes dans l'eau ${ }^{58}$. Mais la transition ne me convenait pas. Il fallait prendre le temps : tu finis [la première action avec l'archet] et tu sais très bien ce qui vient après : là - avec ce but en tête - tu effectues [l'action suivante]. Tu la connais et tu l'amènes précautionneusement. [...] Tu dois prendre le temps.

Le couple historique geste technique/son émis se renverse, et c'est ce qui provoque là un questionnement du point de vue de l'interprétation : comment gérer un geste en soi, non émetteur, ou émetteur avec d'autres sous-entendus, ou bien faire en sorte que ce geste ne soit pas qu'un mouvement technique, un simple geste de contrôle, mais qu'il soit doté d'une valeur pour lui-même?

L'entrée en scène ici est déterminante, car le dépliement dont parle Jean-Loup Rivière ${ }^{59}$ prend un sens différent selon la forme de la pièce musicale envisagée. Dans les pièces musicales classiques et romantiques, il existe une cohérence avec des effets «dramatiques » purement musicaux - l'apparition de la résolution d'un accord, ou la réexposition d'un thème par exemple - qui exigent effectivement que l'on se conçoive un monde. Il est alors nécessaire d'être "habité » de ce mouvement général qui est dessiné par la forme musicale (une suite de forces, de rythmes, de lignes, de dynamiques) :

Qu'il rationalise son acte, qu'il s'appuie sur une représentation imaginaire, qu'il se réfère à un état émotionnel, l'interprète cherche une adéquation entre cet objet qui lui a été donné par un autre, le compositeur, et son propre corps jouant et accomplissant sa vie intérieure; une intériorité façonnée par l'immersion dans un monde qu'en même temps il construit. ${ }^{60}$

Mais si Gérard Siracusa montre un certain détachement en mettant l'accent non pas sur une altérité de la conscience, mais sur la "mise en disponibilité », c'est peut-être parce qu'il est des pièces qui n'ont plus la même suite logique, qui ne suivent plus le grand archétype Arsis/Thésis, et qui ne fonctionnent plus sous le modèle de la narration aristotélicienne - des pièces où le geste doit être entièrement repensé avant. Ce sont des pièces faites de moments différenciés, contrastés, construites sous le modèle du choc et des transitions instantanées, et le matériau utilisé est en adéquation. Le «monde » dont on parlait plus haut, façonné par une intériorité, est devenu un monde partiel, un plurimonde ou un univers composite. Par exemple, dans Les Sept Crimes de l'amour de Georges Aperghis, le phrasé musical existe toujours mais, reflétant peut-être un esprit du temps présent, il donne à saisir une violence dans le choc des matériaux et des formes. Il devient hétérogène car il est constitué de plusieurs éléments différents : glissando vocal, toux, mouvement de la main sur la gorge, renversement de la tête en arrière. Ces éléments ne valent pas pour eux-mêmes, il ne s'agit pas de leur trouver une logique ou de les unifier par un rôle $e^{61}$ : ils sont autant de nouveaux éléments d'une phrase composée de gestèmes et de sonèmes se conglomérant en des superstructures hybrides. Le véritable enjeu du théâtre musical d'avant-garde se situe dans ce moment de tension: une dialectique de l'éclat, du fragment et de la construction, une dialectique de la forme avortée, en rupture, et de la cohérence des idées. 
Face à de telles œuvres, l'interprète ne peut qu'être "disponible ». Son entrée en scène est écrite, de même que ses diverses interventions, il doit animer son jeu de cette force émotionnelle qui est la sienne, car il sait devoir changer d'état à la seconde, mélanger chant et manducation (la soprano croque une pomme à la fin de son trait dans Les Sept Crimes de l'amour) ou passer d'un instrument à un autre dans Zwei-Mann Orchester ou Acustica, ou parler en jouant dans Sonant 1960/... de Mauricio Kagel : il peut agir alors en performer, c'est-à-dire comme quelqu'un qui se manifeste pour lui-même, « qui offre sa présence sur scène à la contemplation ».

\section{En guise de conclusion : toucher [n'est pas jouer]}

Ces quelques entretiens corroborent l'idée que l'acte de l'interprétation est un acte d'objectivation de la pensée musicale et de l'écoute intérieure, qui est rendu possible par l'exploitation de ses capacités techniques corporelles et par une ignition d'ordre émotionnel :

1. L'œuvre musicale constitue pour l'interprète l'objet visé par sa conscience imageante. 2. c'est dans l'acte intentionnel de la conscience qui vise l'objet que se révèlent la vérité et le sens de l'œuvre. 3. la révélation du sens de l'œuvre révèle simultanément l'interprète lui-même. ${ }^{62}$

Cet acte passe par une lecture analytique informée du texte musical qui forge cette écoute intérieure et qui optimise la coordination des gestes techniques, et qui demande une disponibilité du corps. "Habité », sans «sur-jeu », " traversé » par ce qui est joué mais sans «mystique ", jusqu'à l'« absorbement »: quel que soit le substantif ou l'épithète, ce corps, qui est mis en jeu et au jeu, comme moyen, comme siège des émotions, voire comme symbole social, se transforme lorsqu'il traverse le moment de l'entrée en scène, physiquement et psychiquement. Mais ce moment est pluriel, il n'est pas fixe, se constitue en une superposition de strates, que l'on peut décomposer comme ceci en fonction des différentes périodes et zones de l'entrée en scène, dépendantes de l'instrument, du type de pièce et de son effectif :

41 1. Les moments de plateau :

- La préparation de l'instrument et son contact en situation de jeu, l'appropriation sensorielle du dispositif instrumental et de la salle.

- la parution devant le public, et l'aptitude à se mettre en état de communication, le moment où «l'on sent que le comédien va dire quelque chose ». Rappelons que : «Ce que, a priori, l'on remarque du corps, c'est qu'il est visible au regard des autres. Un sens pour autrui se construit donc à partir de son image et de l'éventuelle mise en scène que l'on en fait. Or, si un interprète construit son interprétation avec sa personnalité, [...] il construit aussi son image corporelle, une image qui tisse des liens complexes avec le processus d'interprétation. Cette image corporelle est d'autant plus importante qu'à son tour elle intervient sur la manière d'entendre de l'auditeur $»^{63}$.

- La « mise en place dans le dispositif instrumental $»^{64}$

2. Le Moment musical comme entrée en scène : L'événement sonore spécifique en cours de jeu pour la musique d'ensemble: l'apparition d'une idée musicale nouvelle, d'un instrument, ou d'un moment surchargé d'informations (dynamique, vitesse, nombre de notes par unité de temps) et d'énergie, ou d'un son surprenant ou inattendu ${ }^{65}$, comme un coup de théâtre. 
43 3. Le moment corporel et intérieur : celui de la fabrication de l'« image corporelle » de l'interprète, qui s'inscrit soit dans une altérité, soit dans une concentration intérieure avant le jeu, ou une «mise en disponibilité ». Le compositeur a formulé un système de mouvements et d'énergie - l'œuvre - qui investit le corps de l'interprète. La lecture informée du texte musical établit la structure d'un temps musical et d'une structure discursive des événements sonores qui suivent une force ou un vecteur de projection logico-temporelle dans le temps, tendu vers sa conclusion que l'on peut rapprocher de l'idée de point d'intégration ${ }^{66}$. L'interprète tend son esprit vers le dépliement de ce point d'intégration, il est traversé par cette unité logico-temporelle. Les gestes de son corps sont mus par sa conscience imageante, son intentionnalité sonore, médiatisant le système dynamique de la partition, avec en sus sa propre identité corporelle, son "grain » : deux constructions corporelles qui s'objectivent dans le phrasé à venir.

On le voit, ce qui différencie l'interprète musicien de l'acteur réside dans cet acte de mentalisation du son à venir et dans sa fabrication dans le temps ${ }^{67}$. Or, les théâtres musical et instrumental d'avant-garde remettent en question ce processus. Le moment de plateau et le moment musical sont confondus, le moment intérieur n'est plus celui d'un temps élargi, mais celui d'un temps chronique de l'immédiateté, donnant à voir des fragments : fragments de textes pour Machinations (2000) et Luna Park (2011) d'Aperghis, morceaux d'instruments pour Acustica et Zwei-Mann Orchester de Mauricio Kagel, bribes de voix dans Glossolalie (1961) de Dieter Schnebel, éclats de musiques populaires dans Schwarz auf weiss de Heiner Goebbels. La ligne qui se tendait vers un point d'intégration ou qui était vectorisée dans un système homogène où chaque élément concourrait fonctionnellement à son établissement devient ici plurielle ; ce n'est plus une ligne mais un faisceau, dont les directions tendent à diverger, et dont l'intérêt ne réside plus dans un point d'intégration mais dans de multiples points, une "simultanéité » dotée de lignes de forces, pour reprendre les mots de Hans-Thies Lehmann ${ }^{68}$. Des fragments de sens investissent ainsi toutes les étapes qui constituaient jusque là le propre d'une sorte d'intimité. C'est au sein de cette mise en crise que l'entrée en scène devient un moment complexe et ambigu. Elle se simplifie : l'interprète doit suivre des indications, où plusieurs des moments de l'entrée en scène cités plus haut, qui étaient implicites jusque là, deviennent composés. Mais elle se complexifie : le phrasé qui habitait l'imaginaire de l'interprète est à présent composite, et dans son moment de "mise en disponibilité », il ne tend plus vers une linéarité mais vers la multiplication de moments qualitativement différents. L'interprète n'unifie plus corporellement et socialement un ensemble discret homogène en un phrasé - idéal -, il performe, il agit et fait des actions presque pour elles-mêmes. L'entrée en scène du théâtre musical, paradoxalement, ne peut plus permettre le passage vers la construction d'une quelconque altérité ou d'une fiction - fut-elle abstraite, mais bien au contraire, elle appelle à la décontraction qui précède l'événement performatif au sens littéral, c'est-à-dire l'exploit sportif, et c'est peut-être en cela également que ce genre s'oppose si facilement à d'autres formes de divertissement de concert ou à l'opéra, dont l'appareil a été critiqué dans la première moitié du XXe siècle. Pour le spectateur, ce moment devient un espace de potentialités encore plus grand, celui où l'instrumentiste se montre, mais où il remet en question son rapport aux autres - et le rapport des autres au monde.

On pourrait dire que théâtre et musique ont aujourd'hui échangé leurs perspectives. L'interprète musicien s'est doté en un demi-siècle d'une tradition historique qui lui a permis d'intégrer un representamen dans son geste et dans ses actes de jeu, tandis que le comédien a acquis une forme d'auto-représentation, proche de celle du musicien, où tous 
deux se retrouvent comme performers ${ }^{69}$. Sous cette forme, le musicien se confronterait à un autre symbole. Lui qui jusque là jouait la vie d'un autre - le projet musical de l'œuvre se mettrait à re-jouer sa propre mort, et à affirmer sa vie à lui. Il s'oppose alors à l'acteur car celui-ci «s'il monte sur la scène, c'est rien que pour offrir à l'espace sa disparition »" L'interprète musicien entre en scène en s'affirmant, car il va offrir une expérience singulière de l'existence.

\section{NOTES}

1. René Leibowitz, Le Compositeur et son double, Gallimard, Paris, 1971.

2. René Leibowitz, op. cit., p. 65 (souligné par l'auteur).

3. Voir Giordano Ferrari, Le Théâtre musical d'avant-garde en Italie : Berio, Maderna, Evangelisti, Paris, L'Harmattan, 2001.

4. "Music Theater is theater that is music driven (i.e., decisively linked to musical timing and organization) where, at the very least, music, language, vocalization, and physical movement exist, interact, or stand side by side in some kind of equality but performed by different performers and in a different social ambiance than works normally categorized as operas or musicals »: Eric Salzman et Thomas Desi, The new Music Theater: Seeing the Voice, Hearing the Body, Oxford, Oxford University Press, 2008.

5. Mauricio Kagel, «Sur le théâtre instrumental », Tam-Tam, ed. J.-J. Nattiez, Paris, Christian Bourgois, 1983, p. 105. Voir également Jean-François Trubert, "Dans les coulisses du théâtre instrumental », dans Anne-Sylvie Barthel-Calvet (éd.), Propositions pour une historiographie critique de la création musicale après 1945, Metz, CRULH, 2011, p. 163-195.

6. Cf. Jean-Yves Bosseur, "Théâtre musical», Vocabulaire de la musique contemporaine, Paris, Minerve, 1992.

7. Hans-Thies Lehmann, Le Théâtre postdramatique, trad. Philippe-Henri Ledru, Paris, L'Arche, 2002.

8. Voir à ce propos l'étude récente assez monumentale The Cambridge History of Musical Performance, Colin Lawson et Robin Stowell (éd.), Cambridge, Cambridge University Press, 2012, dont l'ambition est de couvrir les problématiques interprétatives de toutes les périodes historiques, depuis l'antiquité à nos jours. Voir également Nicholas Cook et Mark Everist (éd.), Rethinking Music, Oxford, Oxford University Press, 1999; John Rink (éd.), Musical Performance: a Guide to Understanding, Cambridge, Cambridge University Press, 2002.

9. Jacques Viret (dir.), Approches herméneutiques de la musique, Strasbourg, PUS, 2001. Voir Christian Hauer, « Paroles à l'œuvre par l'œuvre musicale », p. 110-122. Voir également Christian Hauer, «Du sens ou rien », dans Filigrane (2005), n 1, p. 101-113 et enfin Craig Ayrey et Mark Everist (éd.), Analytical strategies and musical Interpretation, Cambridge, Cambridge University Press, 1996.

10. Jane W. Davidson, "The role of the body in the production and perception of solo vocal performance: A case study of Annie Lennox », dans Musicae Scientae (2001), vol. 5 n 2, p. 235-255. Voir également le numéro thématique Perspectives on performance, Musicae Scientae (2005), vol. $9 \mathrm{n}$ -1. Enfin voir Nicholas Cook, «Performance analysis and Chopin's mazurkas », Musicae Scientae (2007), vol. $11 n^{\circ} 2$. Voir également Anthony Gritten et Elaine King (éd.), New Perspectives on Music Gesture, Londres, Ashgate, 2011. 
11. Voir à ce sujet Richard Schechner, Performance Theory, London, New York, Routledge, 2003, également Richard Schechner, Performance Studies, an Introduction, London, Routledge, 2002.

12. Hans-Thies Lehmann, Le Théâtre postdramatique, trad. Philippe Henri-Ledru, Paris, L’Arche 2002, p. 216-217. Voir également les Cahiers d'ethnomusicologie (2008) n² 21 , numéro spécial sur La Performance.

13. Hans-Thies Lehman, op. cit., p. 217.

14. Voir à ce propos l'article assez éclairant d'Andrea Cohen à propos de son spectacle Piano (d)rame dans: Andrea Cohen, "La mise en scène du piano: analyse d'une démarche ", Les Interactions entre musique et théâtre, éd. par Guy Freixe et Bertrand Porot, Montpellier, Ed. L'Entretemps, 2011, p. 60-71.

15. Cf. Nicolas Donin, «Samson François jouant Noctuelles: notes de lecture », dans http:// demeter.revue.univ-lille3.fr/ancien_site.html. Voir également N. Donin, «Problèmes d'analyse de l'interprétation: un essai de comparaison assistée par ordinateur d'enregistrements du premier prélude du Clavecin bien tempéré ", dans Musurgia (2005), vol. XII/4.

16. Joëlle Caullier, "la condition d'interprète », dans la revue en ligne Demeter, consultation à jour le 5 novembre 2012 : http://demeter.revue.univ-lille3.fr/ancien_site.html. Voir également Joëlle Caullier, « La corporéité de l'interprète ", L'Imaginaire musical entre création et interprétation, sous la direction de Mara Lacchè, coll. Univers musical, Paris, L'Harmattan, 2006, p. 133-150.

17. C'est à escient que nous souhaitons rester à la frontière des arts du spectacle. On remarquera que les questions évoquées ici sont par ailleurs abordées sous d'autres formes dans les textes de Valère Novarina, Patrice Pavis, Anne Ubersfeld ou Béatrice Picon-Vallin. Il y a là bien plus qu'une anecdote musicale ou phonatoire, mais un phénomène plus profond lié à l'acte de présence du corps lui-même, que l'on peut aisément retrouver dans certaines chorégraphies de Pina Bausch ou dans les interprétations de Nijinsky. Il est par ailleurs indéniable que certains compositeurs, comme Kurt Weill par exemple, ont tenté de fixer ce « grain » (au sens de Barthes) dans certains songs ou certaines musiques. C'est le cas dans L'Opéra de Quat'sous et dans Mahagonny de Bertolt Brecht, mais cette réalité existe en filigrane dans nombre d'œuvres, y compris de musique pure.

18. Joëlle Caullier, « la corporéité de l'interprète », op. cit., p. 145. Cette idée est à rapprocher de celle développée par Roland Barthes dans ce célèbre article sur « le grain de la voix » : relève du " grain » tout ce qui, à technique et à excellence égales, va différencier un interprète d'un autre, c'est-à-dire, un corps d'un autre corps, un individu (dans sa totalité corporelle façonnée par son expérience propre) d'un autre. Le " grain de la voix » selon Barthes n'a rien à voir avec cette idée répandue en acoustique qui désigne la coloration des harmoniques d'une voix (ses formants) mais plutôt tout ce qui matérialise la spécificité du vécu du corps et de son histoire dans cette voix. Roland Barthes, « Le Grain de la voix », Essais critiques III, L'obvie et l'obtus, Paris, Seuil, 1982, p. 236-245.

19. Reprenant cette idée du toucher, Mine Dogantan-Dack montre comment l'interprète constitue son discours à l'aide de gestes qui ne sont plus destinés à émettre des valeurs de notes discrètes, mais qui unifient une suite composée de plusieurs notes à l'aide d'un seul et même geste devenant une unité. Mine Dogantan-Dack, «In the Beginning was Gesture: Piano Touch and the Phenomenology of the Performing Body", dans New Perspectives on Music and Gesture, op. cit., p. 257. L'auteur tient à remercier Pascal Decroupet pour lui avoir communiqué cette référence.

20. Atelier Théâtre et Musique, fondé en 1976 par Georges Aperghis.

21. Quantité d'exemples peuvent être ajoutés pour illustrer ces questions dans le champ musical qui mériteraient autant de développements ultérieurs. On a vu par exemple la pièce Dialogue de l'ombre double de Pierre Boulez interprétée avec une véritable mise en scène de l'entrée en scène, où le clarinettiste pénètre, dans le noir complet, jouant au milieu de sons répétés par des hautparleurs présents dans toute la salle, jusqu'à prendre sa place sur le plateau - dialectique de la mise en espace, du son réel et du son enregistré, comme une procession rituelle retrouvée en même temps qu'un discret clin d'œil au théâtre. 
22. Picon-Vallin, «Le moment où tout se joue ... », dans Cahiers comédie française, 2001, p. 19.

23. Gérard Siracusa, témoignage épistolaire, questionnaire électronique soumis le 12 septembre 2012. L'auteur souhaite remercier M. Siracusa pour sa coopération et son témoignage.

24. Philippe Serra, entretien oral du 13 septembre 2012.

25. Jean-Loup Rivière, «Entrer en frappant », Cahiers de la Comédie française (2001), n 40, p. 17.

26. Whilhelm Bruck, entretien oral du 7 avril 2011.

27. La définition que Hans-Thies Lehmann reprend à Lyotard du «théâtre énergétique » abonde en ce sens : « [un théâtre] qui ne serait point théâtre de la signification, mais théâtre "des forces, des intensités, des pulsions dans leur présence" ». Hans-Thies Lehmann, Le Théâtre postdramatique ,op. cit., p. 52.

28. Johannes Brahms, $1^{\text {ère }}$ symphonie en do mineur, op. 68.

29. Gérard Siracusa, op. cit.

30. Georges Aperghis, Les sept crimes de l'amour, ed. didascalia, partition en accès libre à télécharger sur le site du compositeur : http://www.aperghis.com/selfservice.html, consulté le 5 novembre 2012.

31. John Cage, Music Walk, Ed. Peter's, 1958. Pour une description des instructions de jeu, voir : http://exhibitions.nypl.org/johncage/node/55, consulté le 5 novembre 2012.

32. Cf. Jean-François Trubert, "Théâtre musical et théâtre instrumental ", dans Théories de la composition au XXe siècle, vol. 2, édité par Nicolas Donin et Laurent Feneyrou, Lyon, Symétrie, 2013 (sous presse), coll. Recherche 20/21.

33. Au sens de Verfremdung, un mot que Kagel a employé dans l'un de ses brouillons de réalisation pour la pièce Antithese. Mauricio Kagel, Antithese, Esquisse, collection Mauricio Kagel, Fondation Paul Sacher, Bâle, Suisse.

34. Michael Praetorius, Syntagma musicum, vol. 2 De organographia, $1^{\text {ère }}$ ed. 1615-1620, réimpr. éditée par Arno Forchert, Kassel, Bärenreiter, 2001.

35. Épreuve de réalisation d'un récital de concert, Auditorium du CNRR de Nice, Licence de Pratiques musicales professionnelles troisième année, programmation artistique de Florian Poirier, interprètes : Florian Poirier et Pierre Andreis, 12 mai 2012.

36. Il était également prévu de procéder à une réalisation de Le Jardin d'en face de Jean-Pierre Drouet, projet qui n'avait pas pu aboutir à ce moment-là.

37. Mauricio Kagel, Match, Mimodrama für Ratsche und Darsteller, Esquisse inédite, Collection Mauricio Kagel, Fondation Paul Sacher, Bâle, Suisse.

38. Mauricio Kagel, Match, Wien, Universal Edition, UE 14543, 1964.

39. Pour la pièce Kerberos pour caisse claire, l'interprète doit dire du texte et ses premiers mots sont : « vous qui entrez, laissez toute espérance », ce qui n'est pas sans entrer en résonance avec certains propos de Valère Novarina qu'on a rappelés ici : « l'acteur qui entre franchit son corps et sa présence, il passe dessous ", dans V. Novarina, "Pour Louis de Funès », Le Théâtre des paroles, Paris, P.O.L., 2007, p. 175.

40. Mauricio Kagel, Match, esquisse inédite, Collection Mauricio Kagel, Fondation Paul Sacher, notre traduction.

41. Louis Jouvet, «L'entrée en scène », dans Les Cahiers de la Comédie française, 2001, vol. 40, p. 15.

42. Gérard Siracusa, témoignage épistolaire, op. cit.

43. Gérard Siracusa, Id.

44. Florian Poirier, entretien du 2 mai 2012, CNRR de Nice.

45. Jean-Loup Rivière, "Entrer en frappant », Les Cahiers de la comédie française, op. cit. p. 17.

46. Entretien oral avec Philippe Serra, 13 septembre 2012.

47. M.-P. Lassus, « Le "toucher intérieur" ", L'imaginaire musical, op. cit., p. 160.

48. Philippe Serra, entretien oral du 13 septembre 2012.

49. Philippe Serra parle de la pièce Psappha (1975) de Xenakis (Note de l'auteur).

50. Florian Poirier, entretien oral, avril 2012. 
51. R. Leibowitz, op. cit., p. 29.

52. R. Leibowitz, Ibid., p. 29-30.

53. Cette conception rejoint également l'idée de la «sur-marionnette» de Craig, Cf. Edward Gordon Craig, De l'art du théâtre, Paris, Ed. Circé, 1998.

54. Entretien oral Florian Poirier du mai 2012, op. cit.

55. À ce sujet, voir le commentaire de Gould lui-même sur sa posture dans: Glenn Gould, Entretiens avec Jonathan Cott, trad. et préface de J. Drillon, Paris, J.-C. Lattès, 1983, p. 64-65.

56. Florian Poirier, entretien oral, avril 2012.

57. Whilhelm Bruck, entretien oral du 7 avril 2011. Nous traduisons.

58. Nous traduisons. Whilhelm Bruck décrit un passage de Zwei-Mann Orchester.

59. " Mettre en scène, c'est déplier » : Jean-Loup Rivière, « Entrer en frappant », op. cit., p. 17.

60. Joëlle Caullier, op. cit., L'Harmattan, p. 148.

61. Andrea Cohen dira «À propos du rôle du pianiste sur scène, je voudrais signaler que l'interprète ne compose pas un personnage ; il reste en premier lieu un instrumentiste » : Andrea Cohen, op. cit., p. 65.

62. (Le compositeur et son double, p. 28, 1971, édition 86).

63. Joëlle Caullier, op. cit., p. 139.

64. Gérard Siracusa, op. cit.

65. À chaque fois, le lecteur aura à cœur de bien considérer qu'un élément sonore existe moins per se que dans sa relation à l'ensemble de l'œuvre musicale, de la même façon qu'un personnage se conçoit dans la logique de l'ensemble de la pièce. Une note de musique n'est inattendue que parce que les structures musicales discursives l'ont jusque-là volontairement occultée, et ont en quelque sorte préparé ce moment de surprise, cette entrée en scène de l'idée musicale. Il reste à ce moment-là à considérer si un événement sonore inattendu possède une fonction structurelle : si il peut-être un accident - et donc n'avoir aucune incidence sur le déroulement par ailleurs - ou bien une rupture - c'est-à-dire donner le signal d'un changement brutal de configurations structurelles et par là se rapprocher du « coup de théâtre ».

66. Selon la définition de Patrice Pavis, d'après Klotz : « Moment où les diverses lignes de l'action [...] convergent en une même scène en fin de pièce ». Patrice Pavis, Dictionnaire du théâtre, éd. revue, Paris, Armand Colin, 2002, p. 263.

67. Pour Christian Accaoui, " "Présent" est une partie du temps, mais c'est aussi le nom de la mise en contact du sujet percevant avec l'étant", ce qui lui permet de distinguer le présent chronique du présent ontique, celui-ci étant définit par les propriétés structurelles de l'objet : «l'objet présent - la présence ontique - requiert une durée, un présent épais qui gèle le continuum temps, le cristallise en laps plus ou moins durables ». Cet épaississement du présent est assuré par la cohésion des forces musicales qui habitent l'œuvre dans sa macro-structure. Il serait intéressant de relire certaines pièces du répertoire musical $\mathrm{du} \mathrm{XX}^{\mathrm{e}}$ siècle selon ce critère afin d'en réinterpréter l'esthétique. Christian Accaoui, Le temps musical, Paris, Desclée de Brouwer, 2001, p. 72-74.

68. Hans-Thies Lehman, Le théâtre post-dramatique, p. 137-138.

69. On peut prendre comme exemple l'interprétation de la pièce ?Corporel (1985) de Vinko Globokar par le violoncelliste de l'ensemble intercontemporain Eric-Maria Couturier. Voir http://youtu.be/QtZeUznYIj0, consulté le 7 janvier 2013.

70. Valère Novarina, « Pour Louis de Funès », op. cit., p. 214. 
INDEX

Mots-clés : musique, geste, théâtre musical

\section{AUTEUR}

\section{JEAN-FRANÇOIS TRUBERT}

Jean-François Trubert est maître de conférences à l'Université de Nice-Sophia Antipolis. CTEL (Centre transdisciplinaire d'Épistémologie de la littérature et des arts vivants) EA 6307. Cet article a bénéficié du soutien du programme AGIS (Acquisition des Gestes d'Interprétation en Situation) du Conseil scientifique de l'Université de Nice. 